\title{
Evidencias de desacople físico-biológico en el sistema de surgencia en La Guajira, Caribe colombiano
}

\author{
Evidence of physical-biological mismatch in the Guajira upwelling system, Colombian Caribbean
}

\author{
Jorge Paramo ${ }^{1,2}$, Marco Correa ${ }^{3}$ y Samuel Núñez ${ }^{4}$
}

\begin{abstract}
${ }^{1}$ Grupo de Investigación Ciencia y Tecnología Pesquera Tropical (CITEPT), Universidad del Magdalena, Cra. 32 No. 22-08, Avenida del Ferrocarril, Santa Marta, Colombia.jparamo@unimagdalena.edu.co

${ }^{2}$ Universität Bremen, Leibniz-Zentrum für Marine Tropenökologie (ZMT), Fahrenheitstr. 6, 28359 Bremen, Alemania ${ }^{3}$ Programa Postdoctoral FONDECYT No. 3110173, Departamento de Geofísica, Universidad de Concepción, Barrio Universitario, Concepción, Chile

${ }^{4}$ Programa de Biología, Facultad de Ciencias Básicas, Universidad del Magdalena, Cra. 32 No. 22-08, Avenida del Ferrocarril, Santa Marta, Colombia
\end{abstract}

\begin{abstract}
The horizontal and vertical distribution of temperature, salinity, magnitude of winds and Chl-a concentration were described, during the upwelling season in the Guajira, Colombian Caribbean, through hydrographic in situ and satellite information, showing variability in spatial distribution of Chl-a during the season with the maximum trade winds in the region. In both, the winds were mainly $N$ to NE and favorable to coastal upwelling, although with a higher intensity during February of 2006. The changes in sea surface temperature and salinity allowed for the determination of the existence of three main upwelling nuclei: the coastal area between the Cabo de la Vela and Punta Gallinas (CV-PG), the zone between Puerto Bolivar and the Cabo de la Vela (PB-CV) and the zone between Santa Marta and the Parque Nacional Natural Tayrona (SM-PNNT). At the end of the upwelling season (February 2006), greater winds increase the offshore advection and the depth of the mixed layer, generating a mismatch between the upwelling and the planktonic biomass in the region.
\end{abstract}

Key words: Winds, chlorophyll, satellite

Resumen.- La distribución horizontal y vertical de la temperatura, la salinidad, la magnitud de los vientos predominantes y la concentración de Chl-a durante la época de surgencia en la Guajira, Caribe colombiano fue descrita, a partir de información hidrográfica in situ e imágenes de satélite, mostrando variabilidad en la distribución espacial de la concentración de Chl-a durante el máximo estacional de los vientos alisios en la región. Los vientos predominantes se observaron $\mathrm{N}$ a NE favorables a la surgencia costera, con mayor intensidad durante febrero de 2006. Los cambios en la temperatura y la salinidad superficial permitieron determinar la existencia de tres núcleos principales de surgencia: la zona costera entre el Cabo de la Vela y Punta Gallinas (CV-PG), la zona entre Puerto Bolívar y el Cabo de la Vela (PB-CV) y la zona entre Santa Marta y el Parque Nacional Natural Tayrona (SM-PNNT). Hacia el final de la época de surgencia, el incremento de los vientos parece generar un incremento en la advección costa-afuera y la profundidad de la capa de mezcla, ocasionando un desacople entre la surgencia costera y la biomasa planctónica en la región.

Palabras clave: Vientos, clorofila, satélite

\section{INTRODUCCIÓN}

El Caribe frente a La Guajira (costa norte de Colombia) está principalmente influenciado por el sistema de los vientos alisios del noreste que ejercen amplia influencia sobre el clima y la variabilidad oceánica. Estos vientos son generalmente intensos a lo largo del año y poseen un efecto desecante, al retirar la humedad ambiental y determinar la baja pluviosidad característica de la región (Andrade 1993, Andrade et al. 2003). La dirección de los vientos se encuentra predominantemente alineada a la línea de la costa y la plataforma continental, lo cual provoca un transporte Ekman fuera de la costa y la surgencia en la zona costera de aguas subsuperficiales (Andrade et al. 2003, Andrade \& Barton 2005). Las aguas bombeadas por el sistema de surgencia de La Guajira son más frías y salinas que las superficiales, y poseen además un mayor contenido de nutrientes que estimula el incremento de la producción biológica y la biomasa fitoplanctónica (Corredor 1979), y de las comunidades de 
macroalgas asociadas a los arrecifes coralinos de la región (Díaz-Pulido \& Garzón-Ferreira 2002). Lonin et al. (2010) afirman que la surgencia costera en la Guajira responde a eventos atmosféricos de modulación estacional forzada por la variación anual de la intensidad de los vientos alisios y a eventos a corto plazo de pocos días que pueden disminuir la intensidad de la surgencia.

Los sistemas de surgencia se caracterizan por tramas tróficas cortas, con pocos taxa intermedios entre el fitoplancton y los principales recursos pesqueros, lo que les confiere una gran importancia económica (Corredor 1979, Paramo et al. 2003, Andrade \& Barton 2005, Richardson 2005, Lalli \& Parsons 2005). La oceanografía local en La Guajira es modulada principalmente por la estacionalidad de la surgencia (Andrade et al. 2003). Se ha observado que estas condiciones determinan la distribución espacial y una fracción importante de la variabilidad de la abundancia del fitoplancton (Caicedo 1975, Duarte 1996, Tigreros 2001, Franco-Herrera \& TorresSierra 2006, Franco-Herrera et al. 2006, Suárez-Villalba et al. 2007, Arévalo-Martínez \& Franco-Herrera 2008) y de las mayores agregaciones de peces pelágicos y demersales de importancia comercial en la región (Paramo \& Roa 2003, Paramo et al. 2003, 2009). Además, los sistemas de surgencia se caracterizan por ser altamente dispersivos, por lo que partículas planctónicas y aguas ricas en nutrientes tienden a ser advectadas fuera de la costa por la deriva inducida por el viento (Roy 1998, Mackenzie 2000), aspecto que podría ser relevante para la entrada de nutrientes y biomasa dentro de la cuenca oligotrófica del Caribe (Franco-Herrera \& Torres-Sierra 2006, García-Hoyos 2008). Sin embargo, recientes observaciones in situ muestran que los máximos en la comunidad planctónica no siempre ocurren durante las épocas de surgencia activa (García-Hoyos 2008), donde las mayores concentraciones no siempre son fácilmente asociables a épocas y zonas de mayor intensidad de vientos. Tales observaciones nos llevaron a plantear la hipótesis que existe un desacople físico-biológico entre los vientos alisios del Noreste favorables a la surgencia y la productividad primaria. De esta forma, es posible encontrar mayores intensidades de los vientos alisios del Noreste en la época de surgencias del Caribe colombiano (diciembre-marzo) asociadas con menores concentraciones de clorofila $a(C h l-a)$. Con el objetivo de conocer las condiciones oceanográficas del sistema de surgencia de La Guajira (Caribe colombiano) se describe la distribución horizontal y vertical de la temperatura, la salinidad, la magnitud de los vientos predominantes y la concentración de $C h l-a$, a partir de información hidrográfica in situ e imágenes de satélite.

\section{Materiales y Métodos}

\section{Área DE ESTUdio}

El área de estudio comprendió la plataforma continental en la región de surgencia de la Guajira, Caribe colombiano, entre Puerto Estrella (PE) y el Parque Natural Nacional Tayrona (PNNT), (12 $\left.44^{\prime} \mathrm{N}-71^{\circ} 41^{\prime} \mathrm{O} ; 1^{\circ} 32^{\prime} \mathrm{N}-74^{\circ} 21^{\prime} \mathrm{O}\right)$, desde $20 \mathrm{~m}$ hasta la isóbata de $\operatorname{los} 200 \mathrm{~m}$ de profundidad. Al norte de la Península de la Guajira la plataforma continental es muy estrecha, presentando profundidades de 200 metros (m) a unas 10 millas náuticas $(\mathrm{mn})$ de la costa y frente a Punta Gallinas (PG) a solo $3 \mathrm{mn}$. Desde el Cabo de la Vela (CV) y hacia el suroeste, la plataforma se ensancha y alcanza un máximo de unas $25 \mathrm{mn}$ frente a Riohacha, luego frente al río Palomino vuelve a reducirse y prácticamente desaparece en el sector del PNNT y Santa Marta (SM) (Fig. 1).

El área está influenciada principalmente por el flujo oeste-noroeste de la Corriente del Caribe (e.g., Wust 1963, Morrison \& Nowlin 1982) y la circulación del giro ciclónico de Panamá (GCP) (Schott \& Molinari 1996, Mooers \& Maul 1998). Las corrientes superficiales del Caribe son forzadas por una activa interacción océano-atmósfera a través del estrés del viento, el flujo de calor, la corriente de borde occidental, inestabilidades de la Corriente del Caribe, y flujo de agua dulce a lo largo de la costa central y sur de América (Andrade et al. 2003). Particularmente, en el sector nororiental del Caribe frente a la costa norte colombiana, la temperatura y salinidad superficial del mar presenta una gran variabilidad anual, registrándose las menores temperaturas y mayores salinidades durante la época seca (diciembre a marzo), cuando los vientos alisios del noreste alcanzan su mayor intensidad $\left(>9 \mathrm{~m} \mathrm{~s}^{-1}\right)$ y la surgencia costera es más intensa (Andrade et al. 2003).

\section{Cruceros oceanográficos}

Las estaciones oceanográficas fueron realizadas en dos cruceros de investigación pesquera abordo del B/I ANCON, del 8 al 15 de diciembre de 2005 (35 estaciones) y del 6 al 16 de febrero de 2006 (51 estaciones) (Paramo et al. 2009) (Fig. 1). En cada estación se tomaron datos hidrográficos de temperatura y salinidad mediante un CTD Sea Bird SB-19. Con esta información se construyeron diagramas T-S basados en la ecuación de estado para el agua de mar y la escala práctica de salinidad (Millero et 


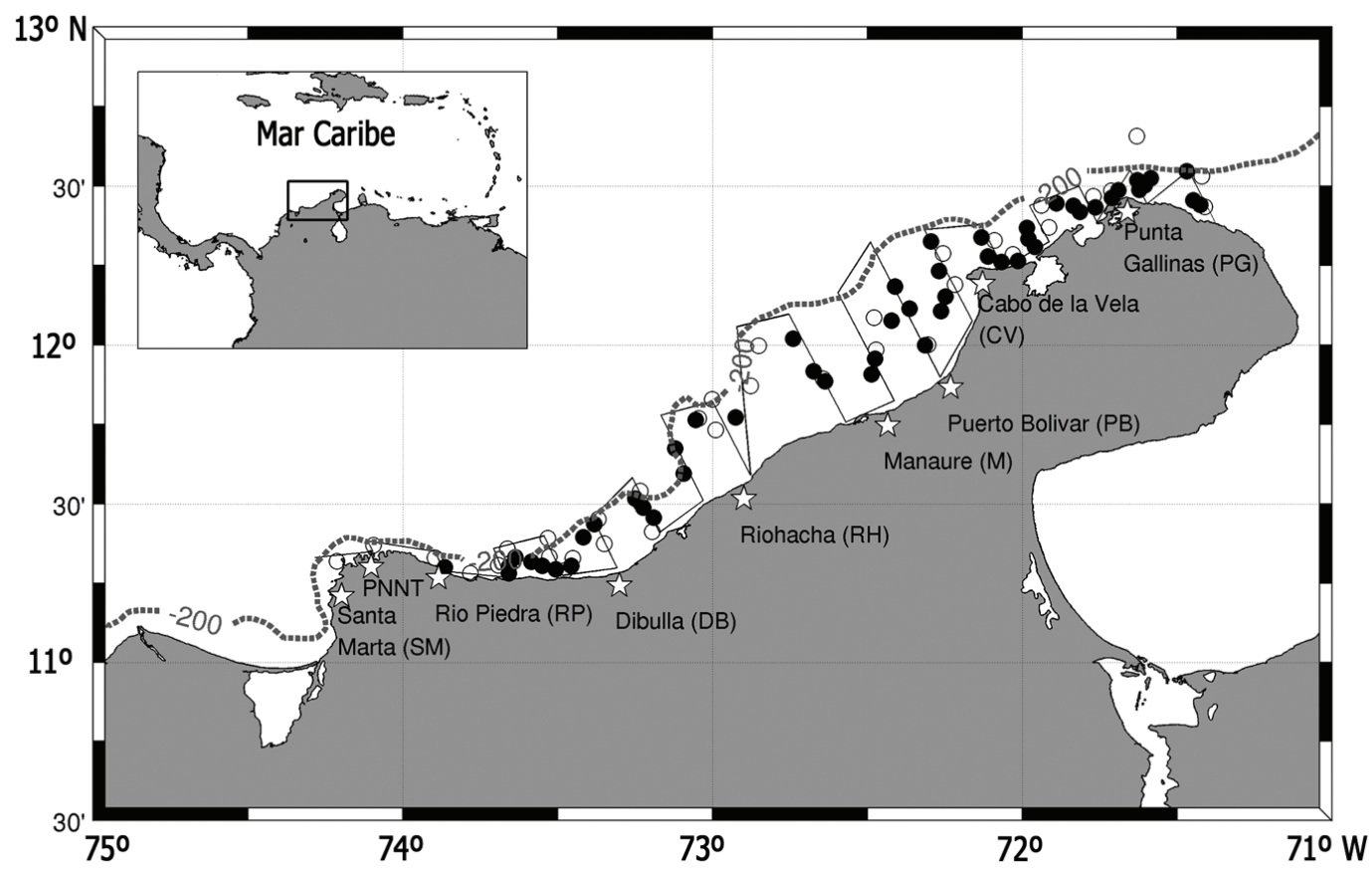

Figura 1. Estaciones oceanográficas (0) y diseño de crucero (-), durante diciembre 2005 (circulo vacío, 35 estaciones) y febrero 2006 (circulo lleno, 51 estaciones) / Oceanographic stations (0) and survey design (-), during December 2005 (empty circle, 35 stations) and February 2006 (full circle, 51 stations)

al. 1980, Millero \& Poisson 1981). Para analizar la distribución de estas variables en superficie, los datos de salinidad, temperatura y densidad a $5 \mathrm{~m}$ de profundidad fueron interpolados a una cuadricula regular de $0,01^{\circ}$ dentro del área de estudio mediante DIVA (DataInterpolating Variational Analysis), una metodología de interpolación basada en funciones ortogonales empíricas que considera el ruido causado por el error de muestreo, la escala espacial típica de la variables analizadas y la topografía de la región (Alvera-Azcarate et al. 2007).

\section{INFORMACIÓN SATELITAL}

Para el periodo correspondiente a los dos cruceros (diciembre de 2005 y febrero de 2006) se obtuvieron el campo promedio-compuesto del viento, la temperatura superficial y la concentración de clorofila a partir de información satelital. La información de viento corresponde a los datos diarios de vientos descendentes
QuickScat L3 de $14^{\circ}$, obtenidos del Jet Propulsión Laboratory SeaWinds Project ${ }^{1}$. Debido a la resolución de las mediciones por satélite, no es posible obtener mediciones satelitales de viento en los primeros $100 \mathrm{~km}$ de la costa, donde se desarrolló la mayor parte de los cruceros. La temperatura proviene de la información diaria MODIS-Aqua, obtenida del Ocean Color $\mathrm{Web}^{2}$. Las clorofila corresponde al producto combinado HERMESGSM de los sensores MODIS-Aqua, MERIS, SeaWiFs, con $\sim 4 \mathrm{~km}$ de resolución, obtenido del GlobColour ${ }^{3}$.

\section{Resultados}

\section{VIENTO EN EL PERIODO 2005-2006}

Las mediciones durante el crucero de diciembre de 2005 mostraron una mayor intensidad de los vientos alisios en el norte del área de estudio ( $\geq 9,0 \mathrm{~m} \mathrm{~s}^{-1}$ en PG) y en el sector de Riohacha $\left(8,0-10,0 \mathrm{~m} \mathrm{~s}^{-1}\right)$, y se registró menor

$1<$ http://podaac.jlp.nasa.gov/quickscat>

${ }^{2}<$ http://oceancolor.gsfc.nasa.gov/ftp.html>

${ }^{3}<$ http://hermes.acri.fr > 
intensidad del viento $\left(2,0 \mathrm{~m} \mathrm{~s}^{-1}\right)$ en el sur entre el río Palomino y el PNNT. Durante el crucero de febrero de 2006, se registraron mayores intensidades en el viento respecto a diciembre de 2005 , principalmente en la zona norte frente al CV $\left(\geq 11,8 \mathrm{~m} \mathrm{~s}^{-1}\right)$ y en el sur frente a la desembocadura del río Palomino $\left(\geq 11 \mathrm{~m} \mathrm{~s}^{-1}\right)$ (Fig. 2a). Sin embargo, el campo de viento satelital fuera de la zona costera muestra la misma tendencia que los vientos registrados en los cruceros: vientos predominantes norte y noreste, con mayor intensidad durante febrero de 2006 $\left(\sim 11 \mathrm{~m} \mathrm{~s}^{-1}\right)$ respecto a los vientos de diciembre de 2005 $\left(\sim 9 \mathrm{~m} \mathrm{~s}^{-1}\right)$. Durante diciembre de 2005 la mayor intensidad de los vientos se observa al norte de $\operatorname{los} 12^{\circ} \mathrm{N}$ y al oeste de los $73^{\circ} \mathrm{W}$. Durante febrero de 2006 los vientos de mayor intensidad se presentan al sur alrededor de los $12^{\circ} \mathrm{N}$, con mayor cercanía a la zona costera. En este mes los vientos poseen un mayor componente norte en su dirección, por lo cual se observan mas alineados a la costa respecto a los vientos en diciembre de 2005 .

\section{TeMPeratura SUPERFICIAL DEL MAR EN DICIEMBRE DE} 2005 - FEBRERO DE 2006

La temperatura superficial del mar satelital (Fig. 2b) y la observada a través de mediciones CTD a $5 \mathrm{~m}$ de profundidad (Fig. 3a), mostraron una alta correspondencia y similar distribución espacial. Tanto en diciembre de 2005 como en febrero de 2006, la zona más cercana a la costa presentó bajas temperaturas superficiales $\left(<27^{\circ} \mathrm{C}\right)$, alta salinidad y densidad (Fig. 3a-c). Sin embargo, entre los dos periodos se observó diferencias en la distribución horizontal de la temperatura superficial: durante el periodo de diciembre de 2005 las aguas de baja temperatura se observaron restringidas a los primeros $200 \mathrm{~km}$ de la costa y conformando varios núcleos a lo largo de la costa, a diferencia del periodo de febrero de 2006 en el cual las bajas temperaturas superficiales se extendieron mas allá de los $600 \mathrm{~km}$ de la costa de una forma más uniforme.

En diciembre de 2005 resaltan 3 núcleos de bajas temperaturas $\left(<24^{\circ} \mathrm{C}\right)$ en la zona costera: la zona comprendida entre el Cabo de la Vela y Punta Gallinas (CV-PG), la zona entre Puerto Bolívar y el Cabo de la Vela (PB-CV) y al zona entre Santa Marta y el Parque Nacional Natural Tayrona (SM-PNNT). De estas, las zonas ubicadas al norte de la Península de La Guajira (PB-CV y $\mathrm{CV}-\mathrm{PG}$ ) presentaron las menores temperaturas superficiales. La dirección de los vientos incidentes las zonas de baja temperatura se observa principalmente paralela a la orientación de la línea de costa $\left(\sim 30^{\circ}\right)$, a excepción de la costa en la zona PB-CV, que se encuentra ubicada en la zona de sotavento del Cabo de la Vela. Durante febrero de 2006 (Fig. 2b y 3a) los núcleos de aguas frías ya no son distinguibles en la zona costera, debido a que las aguas de baja temperatura presentan una mayor extensión superficial y afuera de la costa en toda la región, incursionando mas allá de los $600 \mathrm{~km}$ de la costa y causando una disminución de la temperatura promedio en la mayor parte de la cuenca del Caribe.

\section{Perfiles de Temperatura y SAlinidad y diagramas T-S}

Los perfiles de temperatura y salinidad durante el crucero de diciembre de 2005 (Fig. 4a-c) en Río Piedra (Fig. 4a), Riohacha (Fig. 4b) y Cabo de la Vela (Fig. 4c) muestra la capa de mezcla más somera en aproximadamente $20 \mathrm{~m}$ de profundidad, con respecto al crucero de febrero de 2006 (Fig. 4d, e y f), donde se encuentra más profunda la capa de mezcla, casi a $40 \mathrm{~m}$ de profundidad. Los diagramas de temperatura-salinidad calculados a partir de los perfiles hidrográficos realizados, mostraron la presencia de dos masas de agua en la zona estudiada. Durante el crucero de diciembre de 2005, cerca a la superficie $(\sim 30 \mathrm{~m})$ se observaron Aguas Superficiales del Caribe (CSW), con valores de temperatura y salinidad alrededor de $27^{\circ} \mathrm{C}$ y 36 ups, respectivamente. Sin embargo, en el mismo periodo, a profundidades mayores a $30 \mathrm{~m}$ se observaron temperaturas alrededor de $\operatorname{los} 23^{\circ} \mathrm{C}$ y salinidades de 35,8 ups, características del Agua Subtropical Subsuperficial (SUW) (Fig. 5). Durante el crucero realizado en febrero de 2006 se observó poca estratificación en los perfiles hidrográficos, con una capa de mezcla superior a los $40 \mathrm{~m}$ de profundidad en las estaciones más oceánicas. En este periodo solo se observó la presencia de SUW tanto en superficie como en profundidad.

\section{CONCENTRACIÓN DE CLOROFILA Y SURGENCIA}

Las mayores concentraciones de clorofila se observaron en la zona costera en diciembre de 2005 (Fig. 2c), periodo en el cual los vientos favorables a la surgencia tuvieron menor intensidad. En este periodo las mayores concentraciones se observaron en la zona SM-PNNT, RP$\mathrm{DB}$, entre Riohacha y Manaure (RH-M), entre PB-CV y entre CV-PG. Frente a SM-PNNT y a CV-PG se observaron plumas de alta concentración de clorofila en dirección oeste, con longitudes de 100 y $150 \mathrm{~km}$, respectivamente. En las demás regiones las altas concentraciones de clorofila se distribuyeron a lo largo del borde costero. En la mayor parte del área de estudio, la distribución de altas concentraciones de la clorofila se encontró estrechamente 


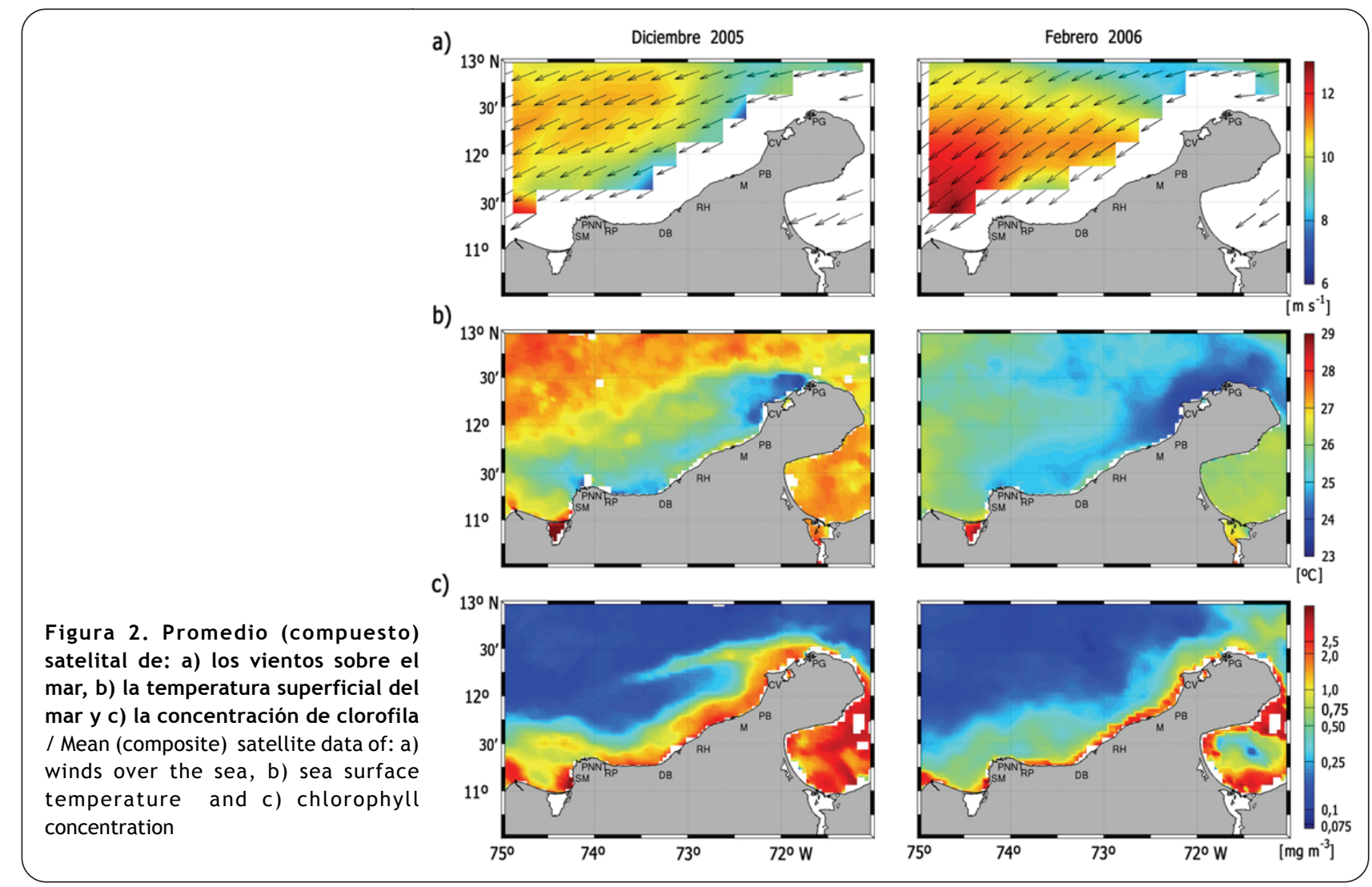

Figura 2. Promedio (compuesto) satelital de: a) los vientos sobre el mar, b) la temperatura superficial de mar y c) la concentración de clorofila temperature and c) chlorophyl concentration

a)

b)
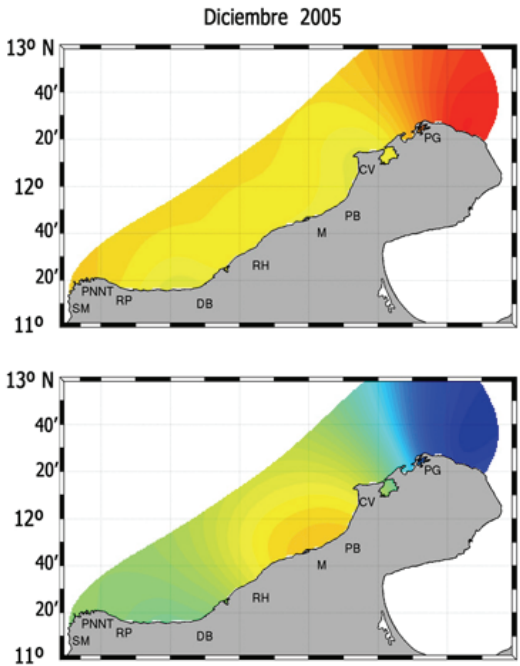

c)

Figura 3. Características de las aguas superficiales en el área de estudio durante diciembre 2005 y febrero 2006. a) temperatura, b) salinidad $y$ c) densidad a $5 \mathrm{~m}$ de profundidad / Characteristics of the surface waters in the study area during December 2005 and February 2006; a) temperature, b) salinity and c) density at $5 \mathrm{~m}$ of depth

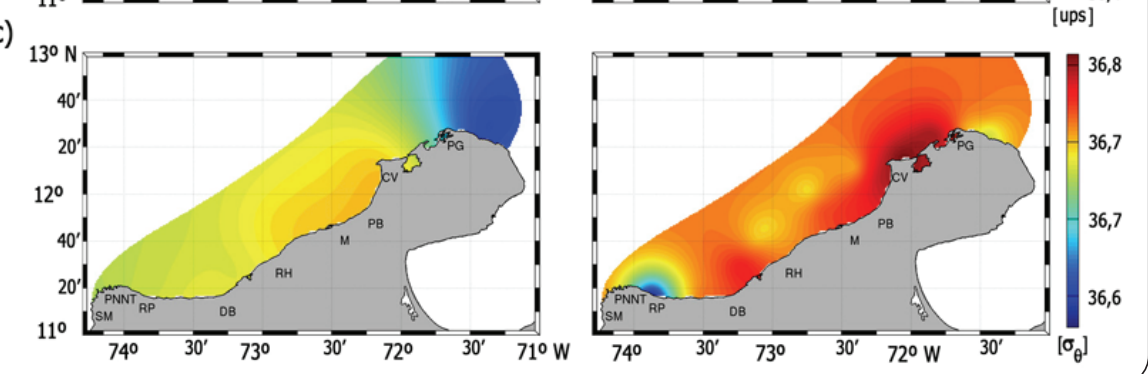

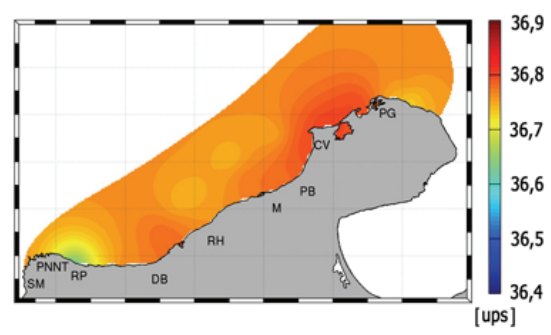




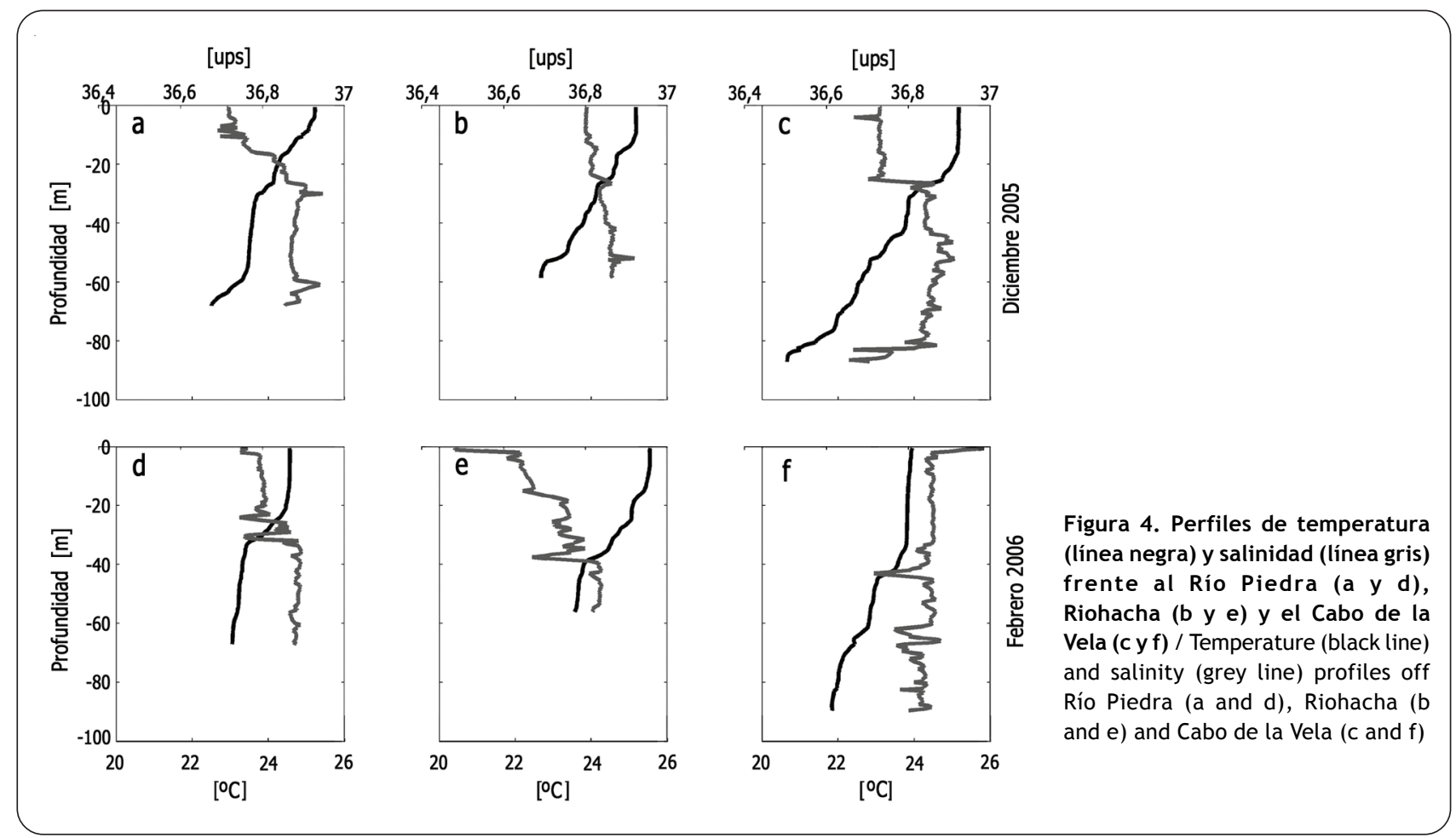

asociada a aguas de baja temperatura. Sin embargo, frente a la zona entre RH-M, las altas concentraciones de clorofila se encontraron asociadas a altas temperaturas superficiales. El mes de febrero de 2006 se caracterizó por presentar menores concentraciones de clorofila satelital en toda la región a pesar de estar sometida a una mayor intensidad en los vientos favorables a la surgencia.

\section{Discusión}

La incidencia de los vientos alisios sobre el mar costero genera un fuerte transporte de Ekman en superficie, lo que conlleva a una intensa surgencia de aguas frías y salinas en la costa que promueve un aumento en la productividad oceánica, principalmente frente de la Guajira (Gordon 1967, Fajardo 1979, Corredor 1979, Corredor 1981, Bula-Meyer 1990, Andrade 1993, Andrade et al. 2003, Andrade \& Barton 2005). La alta intensidad de los vientos alisios observada en este estudio, corresponde con los valores típicos registrados en estudios previos en la región de La Guajira (Andrade 1993, Andrade et al. 2003). Esta alta intensidad junto con su ubicación, le confieren a este sistema características particulares respecto de otros sistemas de surgencia en el mundo. La zona de surgencia de La Guajira se ubica en latitudes bajas (entre 11 y $13^{\circ} \mathrm{N}$ ), al igual que el sistema de surgencia frente a Perú. En ambos sistemas la cercanía con el Ecuador determina un mayor volumen bombeado por la surgencia respecto de otros sistemas ubicados en latitudes medias, debido a la relación inversa entre el transporte de Ekman y el parámetro de Coriolis. Este factor determina que el volumen de la surgencia frente a Perú $(1.6 \mathrm{~Sv})$ sea mayor respecto a los sistemas de surgencia de Canarias, Benguela y California (Tabla 1), aunque la magnitud del viento favorable a la surgencia frente a Perú sea menor (Chávez \& Mesie 2009). La intensidad promedio del viento favorable a la surgencia característica de la Guajira $\left(9,5 \mathrm{~m} \mathrm{~s}^{-1}\right)$ es significativamente mayor que en los otros sistemas de surgencia del mundo. Esto junto con su ubicación tropical, sugiere que La Guajira es el sistema de borde occidental que podría producir el mayor volumen de surgencia por unidad de área en el mundo. Sin embargo, La Guajira presenta una surgencia moderada, con transferencias totales de producción primaria de $3275(\mathrm{t}$ $\mathrm{km}^{-2}$ año ${ }^{-1}$ ) (Criales et al. 2006), en comparación con sistemas como Perú o Benguela ( $>20000 \mathrm{t} \mathrm{km}^{-2}$ año ${ }^{-1}$ ) (Shannon et al. 2003), y en surgencias tropicales como el Golfo de Paria (Venezuela) donde no excede $8000\left(\mathrm{t} \mathrm{km}^{-2}\right.$ año-1) (Mendoza 1993).

A pesar de la alta intensidad de la surgencia en la Guajira, las bajas concentraciones de nutrientes que 
Tabla 1. Características de los principales sistemas de surgencia (modificado de Chávez \& Messié 2009) y del sistema de surgencia de la costa norte de Colombia / Characteristics of the major upwelling systems (modified from Chavez \& Messie 2009) and the upwelling system off the north coast of Colombia

\begin{tabular}{|c|c|c|c|c|c|}
\hline & Benguela & California & NW África & Perú & $\mathrm{CNCC}$ \\
\hline Latitud & $28-18^{\circ} \mathrm{S}$ & $34^{\circ}-44^{\circ} \mathrm{N}$ & $12^{\circ}-22^{\circ} \mathrm{N}$ & $16^{\circ}-6^{\circ} \mathrm{S}$ & $11^{\circ}-13^{\circ} \mathrm{N}$ \\
\hline Velocidad del viento $\left(\mathrm{m} \mathrm{s}^{-1}\right)$ & 7,2 & 7,8 & 6,8 & 5,7 & 9,5 \\
\hline Volumen de la surgencia (Sv) & 1,5 & 1,0 & 1,4 & 1,6 & $\mathrm{~S} / \mathrm{I}^{*}$ \\
\hline Turbulencia $\left(\mathrm{m}^{3} \mathrm{~s}^{-3}\right)$ & 444 & 610 & 371 & 225 & 857,4 \\
\hline$\left[\mathrm{NO}_{3}^{-}\right]$a $60 \mathrm{~m}\left(\mu \mathrm{mol} \mathrm{l} 1^{-1}\right)$ & 16,9 & 11,49 & 19,0 & 16,8 & $<10^{* *}$ \\
\hline Clorofila SeaWifs $\left(\mathrm{mg} \mathrm{m}^{-3}\right)$ & 3,1 & 1,5 & 4,3 & 2,4 & 0,75 \\
\hline
\end{tabular}

*S/I; Sin información en la Bibliografía

Estimación de Corredor (1979)

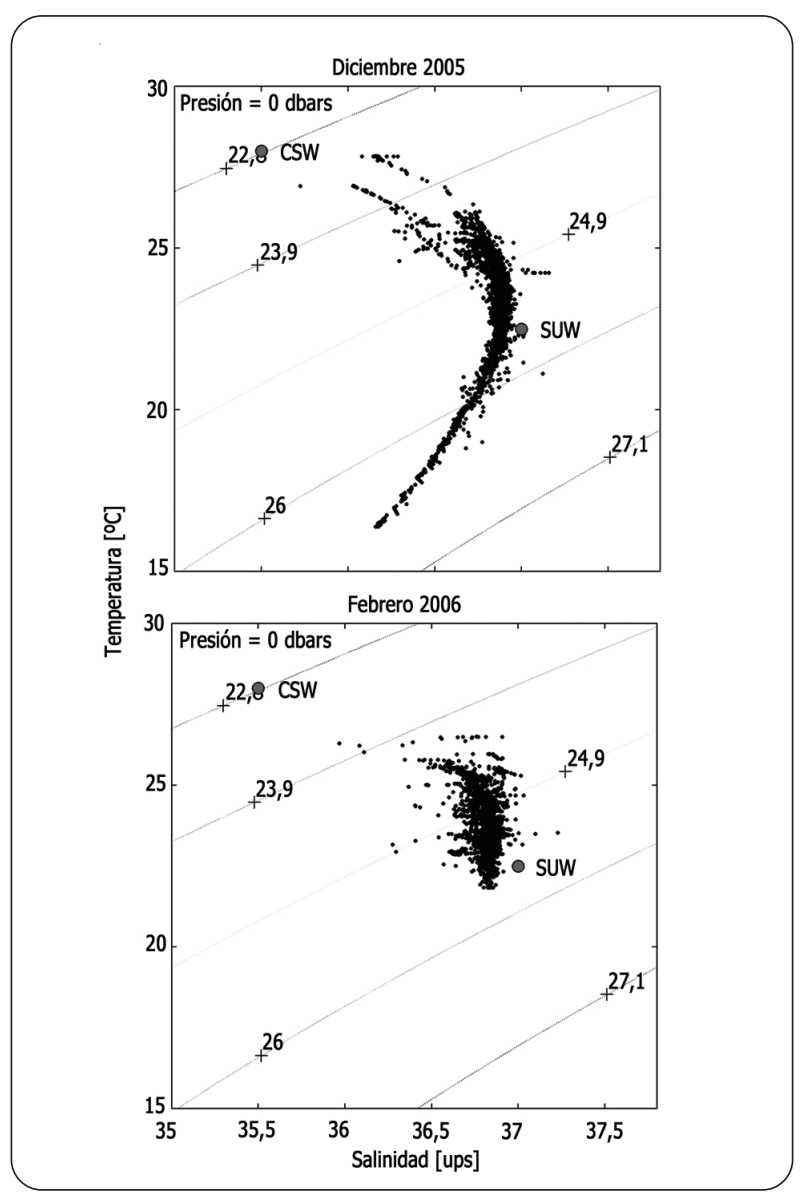

Figura 5. Diagramas T-S (temperatura-salinidad) de las estaciones oceanográficas frente a la costa norte Caribe Colombiano, durante diciembre de 2005 y febrero de 2006. (CSW = Agua Superficial del Caribe, SUW = Agua Subsuperficial Subtropical) / T-S plots for the oceanographic stations off the north Caribbean coast of Colombia, during December 2005 and February 2006. (CSW= Caribbean Surface Water, SUW= Subtropical Subsurface Water) caracterizan el agua que surge en el Caribe parecen determinar las bajas concentraciones de clorofila-a observados en este sistema (Corredor 1979). Las características físicas del agua surgida en la Guajira han sido atribuidas al Agua Subtropical Subsuperficial (SUW) (Wust 1963, Fajardo 1979, Bula-Meyer 1990), que corresponden a una masa de agua que se localiza entre los 100 - $200 \mathrm{~m}$ de profundidad en el Mar Caribe (Hernández-Guerra \& Joyce 2000). Sin embargo, Andrade et al. (2003) sugieren que las aguas subsuperficiales que abastecen el sistema de surgencia en la Guajira pueden estar influenciadas por una rama costera proveniente del Giro de Panamá-Colombia, que fluye a 100 m de profundidad por debajo de la zona de surgencia, cuyo origen superficial y reciente determina la baja cantidad de nutrientes característica del agua que emerge en este sistema.

Los campos de temperatura y densidad superficial revelaron la existencia de una surgencia activa durante los dos periodos de crucero. Durante diciembre de 2005 la surgencia fue de carácter local, restringido a 3 núcleos principales localizados donde la dirección de los vientos se ajusta estrechamente a la orientación de la línea de costa. Mientras que durante febrero de 2006 las aguas surgidas de bajas temperaturas presentaron una mayor extensión en superficie, posiblemente debido a una mayor intensidad de los vientos favorables a la surgencia. Los diagramas T-S revelaron la presencia de aguas de baja densidad en superficie, con propiedades características de SUW, lo cual confirma una intensa surgencia y trasporte costa-afuera durante este periodo. La amplia extensión de las aguas de surgencia en el Caribe ha sido atribuida al transporte de masa producida por las corrientes de deriva que favorece la redistribución de las propiedades físicas 
en la superficie y como consecuencia la ocurrencia de procesos advectivos y difusivos (Fajardo 1979, Andrade \& Barton 2005). En febrero de 2006 las zonas de surgencia ubicadas hacia el norte del área de estudio (PB-CV y CVPG) fueron más amplias e intensas en relación a diciembre de 2005, y se observaron plumas de surgencia de gran extensión que generaron un descenso de la temperatura superficial en la región. Contrariamente, la zona de surgencia ubicada hacia el sur (SM-PT) fue menos intensa durante febrero de 2006. Esto posiblemente debido a la dirección de los vientos que durante esta época tuvo un mayor componente norte en relación a diciembre de 2005 (de 30 a $35^{\circ}$ ), cambio que desfavorece el acople de los vientos con la línea de costa hacia el sur de Dibulla pero incrementa su ajuste hacia el norte. Además, los vientos en la zona sur pueden ser afectados por patrones de recirculación debido a la presencia de la Sierra Nevada de Santa Marta (Fajardo 1979). La diferencia en los vientos incidentes y en la variabilidad de la temperatura superficial sugieren la existencia de una diferente estacionalidad entre la región norte y sur del sistema de surgencia en la Guajira, diferencia que es importante evaluar en mayor detalle para establecer la variabilidad temporal del sistema y su efecto sobre las comunidades biológicas costeras y pelágicas.

En general, las mayores concentraciones de clorofila satelital asociada a aguas surgidas de baja temperatura y alta salinidad se observaron en el periodo de diciembre de 2005, caracterizado por menor intensidad de los vientos. La única excepción fue la zona entre Riohacha y Manaure, que presentó altos valores de clorofila satelital asociados a aguas cálidas. Esta zona presentó una baja intensidad de surgencia durante 2005 por lo que es posible que las mediciones de clorofila satelital pueden estar siendo afectadas por la alta concentración de sedimentos tipo arena media típicamente observada en las aguas costeras de la zona, causada por la interacción entre la poca profundidad de la plataforma, la turbulencia generada por el viento y el aporte de sedimentos de lagunas costeras y ríos. A pesar de que el periodo de febrero de 2006 presentó una mayor intensidad de los vientos favorables a la surgencia y una mayor presencia de aguas surgidas en superficie, los mayores valores de clorofila satelital se observaron en diciembre de 2005, asociados generalmente a aguas de baja temperatura en los núcleos de surgencia de la zona costera y sobre plumas de surgencia.

Es conocido que los cambios latitudinales y estacionales en la productividad primaria resultan de las diferencias en la luz y la disponibilidad de nutrientes. Estos factores físicos determinan la máxima producción posible de fitoplancton (Lally \& Parsons 1997, Greene 2004). Cuando la productividad primaria aumenta, es generalmente acompañada por incremento en la abundancia de fitoplancton. En algunas áreas el zooplancton puede alimentarse de fitoplancton, tan rápido como éste se produce. En la zona tropical del Atlántico y en especial en el Mar Caribe, el zooplancton es menos eficiente en seguir el ritmo de incremento en la producción primaria, lo que induce un desfase, de aproximadamente dos meses, en los ritmos de crecimiento del fitoplancton y del zooplancton (Lally \& Parsons 1997, Mann \& Lazier, 2005). Este cambio se observó en diciembre de 2005 y febrero de 2006 al encontrarse diferencias en las concentraciones de clorofila, teniendo en diciembre mayor concentración, mientras que en febrero la concentración fue menor y más cercana a la costa, como consecuencia probablemente del consumo de zooplancton (Lally \& Parsons 1997). El desacople observado entre la concentración de la clorofila y surgencia costera durante el periodo de mayor intensidad de los vientos (febrero de 2006) puede estar asociado también al incremento de la advección fuera de la costa de aguas surgidas y parches de fitoplancton (Cury \& Roy 1989), o al incremento de la mezcla vertical hasta niveles que restringen el aprovechamiento pleno de los nutrientes por la comunidad fitoplanctónica. Ambos procesos pueden ser provocados por la intensificación de los vientos observada durante este periodo. Este desacople físicobiológico que no ha sido documentado en estudios previos resalta la necesidad realizar más investigación que permita comprender la relación entre las variaciones en las condiciones oceanográficas con los procesos biológicos.

\section{Agradecimientos}

Los autores agradecen al Instituto Colombiano para el Desarrollo de la Ciencia y la Tecnología 'Francisco José de Caldas' (COLCIENCIAS), a la Universidad del Magdalena, al Instituto Colombiano de Desarrollo Rural (INCODER), al Instituto de Investigaciones Marinas y Costeras 'José Benito Vives de Andréis' (INVEMAR), al Institut de Recherche pour le Developpment, France (IRD), por el apoyo financiero, técnico y logístico para el desarrollo del proyecto 'Las Áreas Marinas Protegidas (AMPs): Una Herramienta de Manejo para las Pesquerías Demersales en la Zona Norte del Caribe Colombiano' código 020309-16652. Agradecemos a la Dra. Luisa 
Espinosa (INVEMAR) por sus valiosos comentarios en el manuscrito. Durante la elaboración del artículo Jorge Paramo fue financiado por una beca del Programa ALECOL/DAAD en la Universidad de Bremen (Alemania).

\section{LiTERATURA CITADA}

Alvera-Azcarate A, A Barth, JM Beckers \& RH Weisberg. 2007. Multivariate reconstruction of missing data in sea surface temperature, chlorophyll, and wind satellite fields. Journal of Geophysical Research Oceans 112: C03008, 11 p.

Andrade C. 1993. Análisis de la velocidad del viento en el mar Caribe. Boletín Científico Centro de Investigaciones Oceanográficas e Hidrográficas 13: 33-43.

Andrade CA, ED Barton \& CHNK Mooers. 2003. Evidence for an eastward flow along the Central and South American Caribbean Coast. Journal of Geophysical Research 108: 1-11.

Andrade C \& E Barton. 2005. The Guajira upwelling system. Continental Shelf Research 25: 1003-1022.

Arévalo-Martínez D \& A Franco-Herrera. 2008. Características oceanográficas de la surgencia frente a la Ensenada de Gaira, Departamento del Magdalena. Boletín de Investigaciones Marinas y Costeras 37(2): 131-162.

Bula-Meyer G. 1990. Oceanografía. En: Jimeno MC (ed). Caribe de Colombia, pp. 135-153. Fondo José Celestino Mutis, Bogotá.

Caicedo IE. 1975. Productividad primaria y fitoplancton en la Bahía de Neguange (Parque Nacional Natural Tayrona). Anales del Instituto de Investigaciones Marinas Punta de Betín 9: 7-14.

Chavez FP \& M Messié. 2009. A comparison of Eastern Boundary Upwelling Ecosystems. Progress in Oceanography 83: 80-96.

Corredor JE. 1979. Phytoplankton response to low level nutrient enrichment through upwelling in the Colombian Caribbean basin. Deep Sea Research A 26: 731-741.

Corredor JE. 1981. Apuntes sobre la circulación costera en el Caribe noroccidental colombiano. Boletín Científico Centro de Investigaciones Oceanográficas e Hidrográficas 3: 3-8.

Criales MI, CB García \& M Wolff. 2006. Flujos de biomasa y estructura de un ecosistema de surgencia tropical en La Guajira, Caribe colombiano. Revista de Biología Tropical 54(4): $1257-1282$.

Cury P \& C Roy. 1989. Optimal environmental window and pelagic fish recruitment success in upwelling areas. Canadian Journal of Fisheries and Aquatic Sciences 46: 670-680.

Díaz-Pulido G \& J Garzón-Ferreira. 2002. Seasonality in algal assemblages on upwelling-influenced coral reefs in the Colombian Caribbean. Botánica Marina 45: 284-292.
Duarte G. 1996. Estructura del fitoplancton al noroeste de la Guajira, durante el crucero Caribe 1-93. Trabajo de Biología Marina, Universidad de Bogotá Jorge Tadeo Lozano, Santa Marta, 98 pp.

Fajardo G. 1979. Surgencia costera en las proximidades de la península colombiana de La Guajira. Boletín Científico Centro de Investigaciones Oceanográficas e Hidrográficas 2: 7-19.

Franco-Herrera A. 2008. ¿Hacia dónde camina la investigación del plancton colombiano? Revista Innovación y Ciencia 15(1): 54-57.

Franco-Herrera A, L Castro \& PC Tigreros. 2006. Plankton dynamics in the South-Central Caribbean sea: Strong seasonal changes in a coastal tropical system. Caribbean Journal of Science 42(1): 24 -38.

Franco-Herrera A \& EA Torres-Sierra. 2006. La comunidad fitoplanctónica en el evento de surgencia frente al mar Caribe centro de Colombia. Actualidades y Divulgación Científica 10(1): 159-172.

García-Hoyos LM. 2008. Dinámica en el acoplamiento océanoatmósfera y su influencia en la biomasa fitoplanctónica durante un evento de surgencia y no surgencia en la zona costera del departamento del Magdalena. Tesis de Biología Marina, Universidad de Bogotá Jorge Tadeo Lozano, Santa Marta, 130 pp.

Gordon AL. 1967. Circulation of the Caribbean Sea. Journal of Geophysical Research 72: 6207-6223.

Greene T. 2004. Marine science, marine biology and oceanography, 623 pp. AMSCO, New York.

Hernandez-Guerra A \& TM Joyce. 2000. Water masses and circulation in the surface layers of the Caribbean at $66^{\circ} \mathrm{W}$. Geophysical Research Letters 27: 3497-3500.

Lalli C \& T Parsons. 1997. Biological oceanography: An introduction, $320 \mathrm{pp}$. Butterworth-Heinemann, Oxford.

Lonin SA, JL Hernández \& DM Palacios. 2010. Atmospheric events disrupting coastal upwelling in the southwestern Caribbean. Journal of Geophysical Research 115 DOI: 10.1029/2008JC005100.

Mackenzie BR. 2000. Turbulence, larval fish ecology and fisheries recruitment: a review of field studies. Oceanologica Acta 23: 357-375.

Mann K \& J Lazier. 2005. Dynamics of marine ecosystems: Biological-physical interactions in the oceans, $512 \mathrm{pp}$. Wiley-Blackwell, Oxford.

Millero FJ \& A Poisson. 1981. International one-atmosphere equation of state of seawater. Deep Sea Research A 28: 625-629.

Millero FJ, CT Chen, A Bradshaw \& K Scleicher. 1980. A new high-pressure equation of state for seawater. Deep Sea Research A 27: 255-264. 
Mooers CNK \& GA Maul. 1998. Intra-Americas sea circulation. En: Robinson AR \& KH Brink (eds). The Sea 11: 183-208. John Wiley, New York.

Morrison JM \& WD Nowlin Jr. 1982. General distributions of water masses within the Eastern Caribbean Sea during the winter of 1972 and fall of 1973 . Journal of Geophysical Research 87: 4207-4229.

Paramo J \& R Roa. 2003. Acoustic-geostatistical assessment and habitat-abundance relations of small pelagic fish from the Colombian Caribbean. Fisheries Research 60: 309-319.

Paramo J, RA Quiñones, A Ramírez \& R Wiff. 2003. Relationship between biomass density of small pelagic and environmental factors in the Colombian Caribbean Sea: an analysis based on hydroacustic information. Aquatic Living Resources 16(3): 239-245.

Paramo J, L Guillot-Illidge, S Benavides, A Rodríguez \& C Sánchez-Ramírez. 2009. Aspectos poblacionales y ecológicos de peces demersales de la zona norte del Caribe colombiano en relación con el hábitat: una herramienta para identificar áreas marinas protegidas (AMPs) para el manejo pesquero. Caldasia 31(1): 123-144.

Richardson PL. 2005. Caribbean Current and eddies as observed by surface drifters. Deep-Sea Research II 52: 429-463.
Roy C. 1998. Upwelling-induced retention area: a mechanism to link upwelling and retention processes. South African Journal of Marine Science 19: 89-98.

Schott FA \& RL Molinari. 1996. The western boundary circulation of the subtropical warmwatersphere. En: Krauss W (ed). The Warmwatersphere of the North Atlantic Ocean, pp. 229-252, Gebrueder Bontraeger, Berlin.

Suarez-Villalba V, A Franco-Herrera \& ML Cañón. 2007. El microfitoplancton en los principales muelles de la Bahía de Cartagena, Caribe colombiano, vectores posibles de floraciones microalgales. Boletín Científico Centro Investigaciones Oceanográficas Hidrográficas 25: 135-149.

Tigreros P. 2001. Biodiversidad y valoración bioquímica del fitoplancton marino en ambientes costeros mesotróficos y oligotróficos tropicales, Caribe colombiano. Tesis de Biología Marina, Universidad de Bogotá Jorge Tadeo Lozano, Bogotá, 173 pp.

Wust G. 1963. On the stratification and the circulation in the cold water sphere of the Antillean-Caribbean basin. Deep Sea Research 10: 165-187.

Recibido el 13 de junio y aceptado el 11 de noviembre 